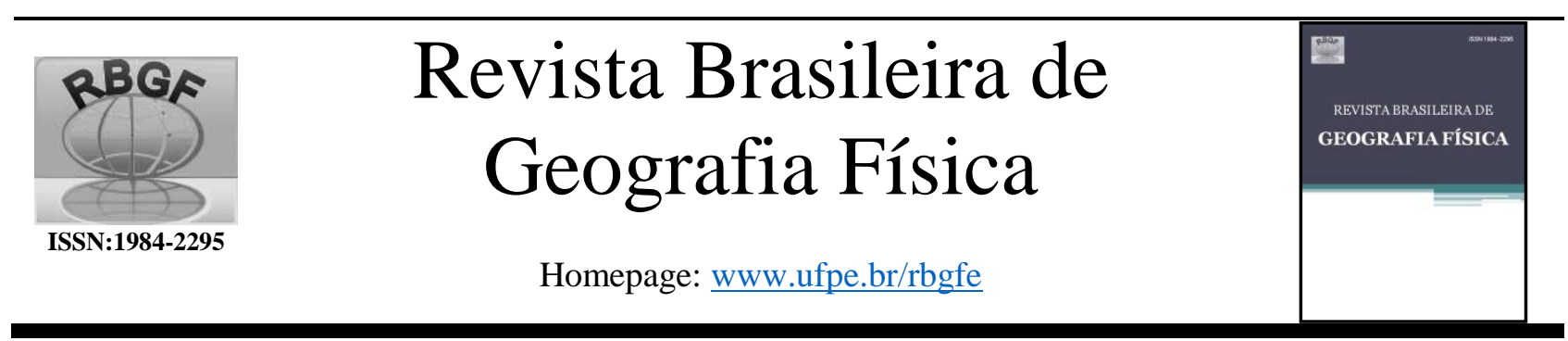

\title{
Métodos de interpolação espacial do regime pluviométrico no espaço urbano de Catalão, Goiás $^{1}$
}

\author{
Thiago Patrocínio da Silva²; Rafael de Ávila Rodrigues ${ }^{3}$; Rafael Coll Delgado ${ }^{4}$; Leydimere Janny Cota \\ Oliveira $^{5}$; Antover Panazzolo Sarmento ${ }^{6}$
}

${ }^{2}$ Mestre em Geografia, UFG, Catalão, GO (Thiago.patrocinio@ gmail.com). ${ }^{3}$ Professor da Unidade

Acadêmica Especial Instituto de Geografia, Catalão (GO) (UFG) (rafael.avila.roodrigues@gmail.com).

${ }^{4}$ Professor do Departamento de Ciências Ambientais, Instituto de Floretas (DCA/IF/UFRRJ).

rafaelcolldelgado32@ gmail.com (autor correspondente). ${ }^{5}$ Professora do Campus Itaqui, Itaqui, RS (UFP)

(leydimereoliveira@ unipampa.edu.br) e ${ }^{6}$ Professor do Curso de Engenharia Civil da Universidade Federal de Goiás - Regional Catalão (UFG/RC) (antoverps@gmail.com).

Artigo recebido em 13/09/2017 e aceito em 29/03/2018

\begin{abstract}
R E S U M O
O presente estudo visa à realização de um estudo de métodos de interpolação espacial que melhor se ajustem a área urbana de Catalão, Goiás. Foi avaliado o desempenho espacial da chuva coletada por uma rede de pluviômetros modelo "Ville de Paris" através de três modelos: Exponencial, Gaussiano e Esférico no período de 2015 a 2016. Utilizaram-se dados diários de chuva dos pluviômetros e estações automática e convencional do Instituto Nacional de Meteorologia. Para validação foram adotados os seguintes parâmetros: regressão linear e correlação linear (Pearson) ( $r^{2}$ e r), ajustamento de curvas, seguido do Erro Padrão de Estimativa (EPE), índice de concordância (d) e Viés Médio (VM). O ano de 2015 foi o mais inconsistente pontualmente, onde, o $\mathrm{r}^{2}$ variou de $93 \%$ comparado com a convencional a $64 \%$ com a automática. Os erros foram maiores neste ano com VM para convencional de -146 mm e EPE de 21,13 mm, já a automática os erros foram menores com VM de 13,41 mm e EPE de 49,72 mm. Para o ano de 2016 o r $^{2}$ foi superior a 95\% para ambas estações. O índice d foi igual a 1 para ambas estações. O melhor GDE encontrado foi para o ano de 2016, em que se ajustou um modelo Exponencial. Os piores resultados foram encontrados para o ano de 2015, onde ambos modelos apresentaram Efeito Pepira Puro, o que inviabiliza a utilização de KO. As regiões de maiores e menores adensamentos geográficos influenciaram diretamente nos volumes precipitados em Catalão, Goiás.
\end{abstract}

Palavras Chave: Análise espacial, geoestatística, sinótica.

\section{Methods of spatial interpolation of the pluviometric regime in the urban space of Catalão,} Goiás

\section{A B S T R A C T}

The present study aims to perform a study of spatial interpolation methods that best fit the urban area of Catalão, Goiás. The spatial performance of the rain collected by a network of "Ville de Paris" rain gauges was evaluated through three models: Exponential, Gaussian and Spherical data from 2015 to 2016. Rainfall data were used for rain gauges and automatic and conventional stations of the National Meteorological Institute. For validation, the following parameters were adopted: linear regression and linear (Pearson) correlation $\left(\mathrm{r}^{2}\right.$ and $\mathrm{r}$ ), curve fitting, followed by Standard Error of Estimation (SEE), concordance index (d) and Medium Bias. The year 2015 was the most inconsistent on time, where $\mathrm{r}^{2}$ ranged from $93 \%$ compared to conventional to $64 \%$ with automatic. The errors were larger in this year with conventional MB of $-146 \mathrm{~mm}$ and EPE of $21.13 \mathrm{~mm}$, while the automatic errors were smaller with MB of $13.41 \mathrm{~mm}$ and SEE of 49.72 $\mathrm{mm}$. For the year $2016 \mathrm{r}^{2}$ was higher than $95 \%$ for both seasons. The index $\mathrm{d}$ was equal to 1 for both seasons. The best DSP found was for the year 2016, in which an Exponential model was fitted. The worst results were found for the year 2015, where both models presented Pure Pepira Effect, which made it impossible to use OK. The regions with larger and smaller geographic densities influenced directly the volumes precipitated in Catalão, Goiás.

Keywords: Spatial analysis, geostatistics, synoptic.

Silva, T. P., Rodrigues; R. Á., Delgado, R. C., Oliveira, L. J. C., Sarmento, A. P. 


\section{Introdução}

Sabe-se que as variações climáticas são uma preocupação latente em todo o mundo, bem como que a precipitação pluviométrica configura-se diretamente quanto ao volume hídrico de uma região específica, e tem relevância significativa quanto à caracterização do clima em uma determinada região.

Dessa forma, salienta-se que a precipitação é a variável meteorológica que influencia diretamente no balanço hídrico de uma região, podendo-se por meio dela determinar o excesso ou escassez de chuvas de uma área específica, sendo essencial na constituição do clima de uma determinada extensão territorial, agindo principalmente sobre o balanço de água no solo, na temperatura e umidade do ar (Xavier et al., 2016).

O entendimento tangente à distribuição e comportamento das chuvas no espaço urbano, faz-se necessário, uma vez que, mesmo diante da necessidade, ainda ocorre falta de planejamento e maiores pesquisas sobre o tema, principalmente, no que se refere a pequenas cidades, bem como, pela necessidade de tentar desvendar a possível existência de um padrão normal de distribuição das mesmas. As cidades ao se transformarem com o tempo modificam os elementos do clima (Viana e Amorim, 2009; Santos e Amorim, 2015) estas modificações nos elementos podem ocasionar extremos de chuvas, ilhas de calor e aumento de desastres locais (Pereira, 2016; Zanella e Sales, 2016).

Nas cidades brasileiras, entre os anos de 1970 e 2000, a explosão demográfica aumentou de $56 \%$ para $80 \%$, conforme Instituto Brasileiro de Geografia e Estatística e esse rápido desenvolvimentos populacional da zona urbana, lamentavelmente, é indício de crescimento desordenado e de grandes impactos ambientais e alterações no meio ambiente, com vistas ao clima, principalmente, no que se refere às zonas urbanas.

Neste contexto, sabe-se que as ações antrópicas promovem mudanças na paisagem, e essas, resultam em processos de caos urbano em períodos de fortes chuvas, pois, em função de extensas áreas impermeabilizadas, compactas, com baixa ou quase nenhuma velocidade de infiltração, o volume precipitado não dispõe, muitas vezes, de mecanismos eficazes para realização da drenagem.

Como ocorre em todo o País, na região centro-oeste também existe cidades de pequeno porte, onde os estudos atrelados ao clima são limitados, porém, se torna de grande relevância considerar e avaliar os fatores climáticos desde sua fundação, bem como processo de expansão, na proporção em que a identificação das variações higrométricas e térmicas promova melhores condições ambientais e de vida (Mendonça, 2003).

As mudanças futuras das chuvas em determinadas regiões como o aumento do número de períodos secos durante um ano (Polade et al., 2014) representa uma grave ameaça a produtividade agrícola, o que poderá comprometer a segurança alimentar a nível global. Além disso, o número de desastres ocorridos em algumas localidades do globo em consequência das chuvas pode estar associado por atividades humanas locais (Fengqing et al., 2005).

Desse modo, o conhecimento da sucessão de anos secos e chuvosos, suas anomalias e seus padrões temporais são de extrema relevância para a tomada de decisão quanto ao uso do espaço geográfico, pois com o auxílio da rede pluviométrica se torna possível compreender a dinâmica das chuvas a nível espaço-temporal.

A análise espacial surge como uma das várias formas de se avaliar e compreender os fenômenos das mais diversas áreas de conhecimento e sua correlação a alguns eventos, principalmente os climáticos (Santana et al., 2015). Modelos espaciais utilizando a geoestatística têm sido aplicados para a estimativa espaço-temporal de variáveis, na modelagem de fenômenos e simulações de cenários climáticos (Gondim et al., 2008; Delgado et al., 2014).

Dados espaciais de chuva tem sido amplamente utilizados em todo o globo e servem para subsidiar as pesquisas para o planejamento agrícola, hidrologia entre outros (Sun et al., 2015).

De acordo com Castro et al. (2010), a qualidade de uma interpolação de dados depende da distribuição e do conhecimento dos pontos usados no cálculo, além da correlação de 
Revista Brasileira de Geografia Física v.11, n.03 (2018) 745-757.

modelos estatísticos com os fenômenos em estudo.

São inúmeros os trabalhos recentes que utilizam a interpolação espacial para a variável chuva, onde o método espacial empregado é a Krigagem Ordinária (KO) (Gois et al., 2015; Trindade et al., 2016).

Dessa forma, torna-se prioritário o entendimento da dinâmica das chuvas no espaço urbano, na perspectiva de nortear investimentos públicos e/ou privados, buscando minimizar os impactos negativos de tais alterações no espaço urbano, no que se diz respeito ao planejamento de infraestrutura.

No entanto, ainda não existem trabalhos na cidade de Catalão, Goiás que calculem e estimem o melhor método espacial de chuvas. Com base no que foi apresentado, este trabalho teve por objetivo geral analisar métodos de interpolação espacial das chuvas no espaço urbano de Catalão, assim como contribuir com estudos voltados ao clima local, fornecendo, assim, subsídios a análises ambientais em função do seu crescimento urbano e da evolução histórica da cidade.

\section{Material e métodos}

\section{Área de estudo}

A área de estudo corresponde a área urbana de Catalão, localizada no Sudeste do Estado de Goiás, latitude $18^{\circ} 9^{\prime} \quad 57^{\prime \prime} \mathrm{S}$ e longitude $47^{\circ} 56^{\prime} 47^{\prime \prime} \mathrm{O}$, faz parte, também, do Sudeste Goiano, Mesorregião Sul Goiana (IBGE, 2008), local onde foi realizado o monitoramento pluviométrico de forma integrada para análise da distribuição e gênese das chuvas no espaço urbano, a partir da utilização dos dados coletados por pluviômetros distribuídos ao longo da cidade, por meio de uma rede de pluviômetros modelo Ville de Paris, conforme Figura 1 e Tabela 1.

O município possui estimativa de 100.590 habitantes, conforme estimativa realizada no ano de 2016, perfazendo uma área territorial de $3.821,463 \mathrm{~km}^{2}$. Possui densidade demográfica de 22,67 hab. $\mathrm{km}^{-2}$, e conta com 90\% de urbanização, decorrente do processo de sua evolução, atrelado ao agronegócio, indústria e mineração (IBGE, 2017).

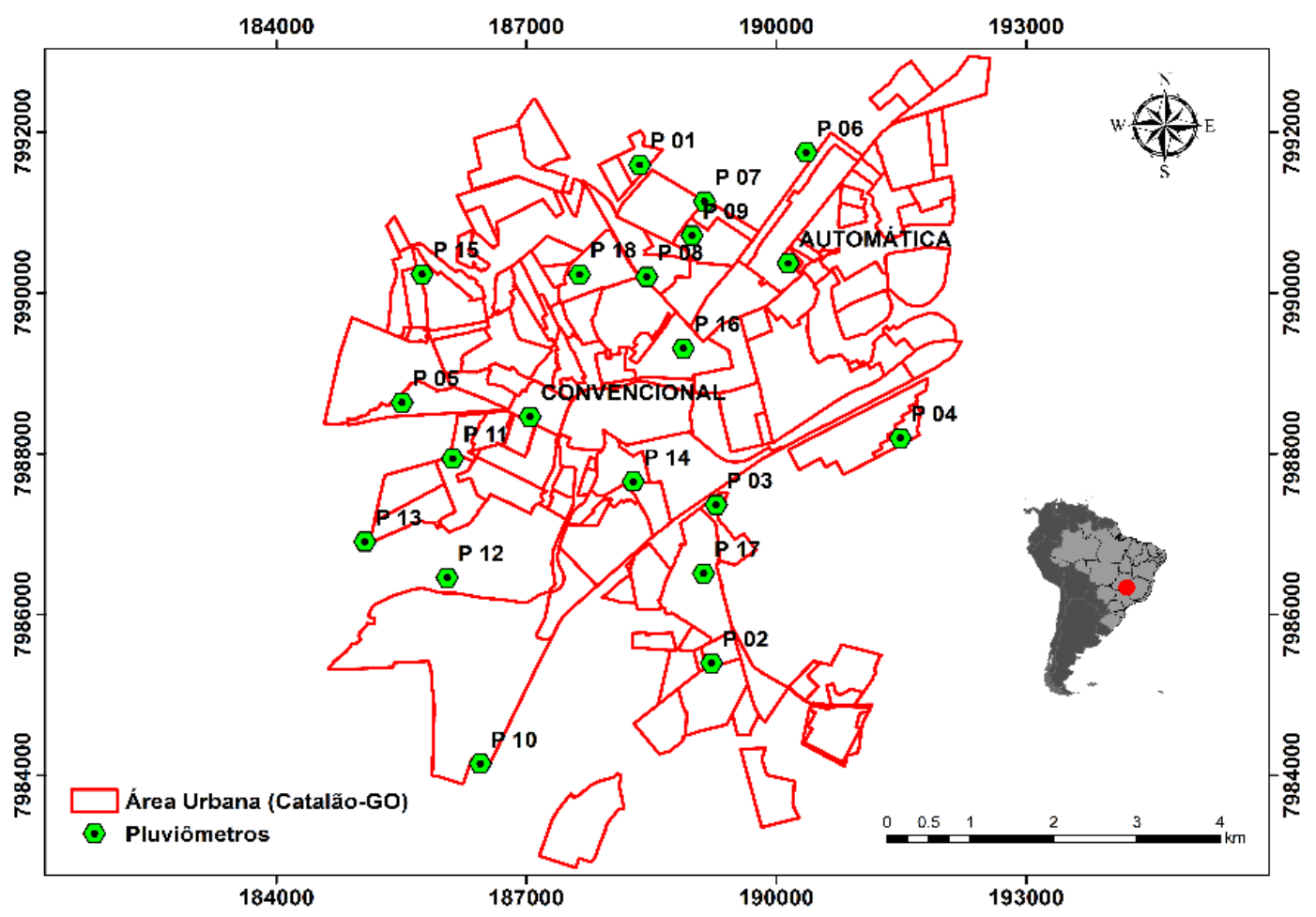

Figura 1. Distribuição espacial dos pluviômetros instalados na área urbana de Catalão-GO. 
Revista Brasileira de Geografia Física v.11, n.03 (2018) 745-757.

Tabela 1. Distribuição espacial e descrição dos pluviômetros instalados na área urbana de Catalão-GO.

\begin{tabular}{cc} 
PLUVIÔMETRO & ENDEREÇO \\
\hline P 01 & Evelina Nour II - Escola Municipal \\
P 02 & Estrela - Reservatório - 500m³ \\
& Castelo Branco II - Reservatórios \\
P 03 & 500m \\
P 04 & Pontal Norte - Reservatório 500m³ \\
P 05 & Escola Municipal Wison da Paixão \\
P 06 & ETA Ipanema \\
P 07 & Elevatória Leblon \\
P 08 & ETA \\
P 09 & Condomínio Residencial Olinda \\
P 10 & ETA - Dimic \\
P 11 & Elto da Boa Vista - Reservatório \\
P 12 & ETE - Dimic \\
P 13 & Escola Municipal Sta. Terezinha \\
P 14 & JK - Labibe Fayad \\
P 15 & Bairro Paineiras - Rua 2006 \\
P 16 & Setor Paraíso \\
P 17 & Jardim Catalão \\
P 18 & Escola CAIC \\
CONVENCIONAL & PIO GOMES \\
AUTOMÁTICA & Universidade Federal de Goiás \\
\hline &
\end{tabular}

\section{Clima}

A cidade de Catalão apresenta temperatura média anual em torno de $22^{\circ} \mathrm{C}$, e a chuva apresenta uma grande variabilidade temporal, principalmente na escala de tempo interanual. As chuvas mais significativas iniciam-se em setembro de cada ano e estendem-se até o início de abril do ano seguinte (INMET, 2015).

Observa-se que, de acordo com o estudo, a menor ocorrência de chuvas no município foi registrada em junho e julho de 2016, com apenas $10,0 \mathrm{~mm}$ e $10,3 \mathrm{~mm}$ de média, respectivamente, ao passo que o maior valor foi registrado em dezembro de 2015, com total médio de $283,5 \mathrm{~mm}$, ressaltando alta variabilidade que pode ser observada no período seco e chuvoso.

Deve-se destacar a precipitação anual do período analisado considerando a média de
COORDENADAS

ALTITUDE
889

871

888

897

866

907

886

930

910

852

866

816

862

900

886

854

867

903

859

$18^{\circ} 9^{\prime} 12.43$ "S $\quad 47^{\circ} 55^{\prime} 42.47^{\prime \prime O} \quad 906$

1.484,8 mm. Mais detalhes sobre a caracterização climática na região Sudeste de Goiás podem ser encontrados em Rodrigues et al. (2009 e 2012).

\section{Coleta de chuva "Ville de Paris"}

Foram levantados os dados pluviométricos de vinte pontos no período compreendido entre 2015 (abril, maio, junho, julho, setembro, outubro e dezembro) a 2016 (janeiro, fevereiro, março, abril e maio) junto à Estação Meteorológica Convencional e Automática do Instituto Nacional de Meteorologia (INMET) e pluviômetros modelo Ville de Paris, para estudar o regime pluviométrico no espaço urbano de CatalãoGO.

Os volumes precipitados foram coletados diariamente às $9 \mathrm{~h}$, conforme orientação do INMET e da Organização 748

Silva, T. P., Rodrigues; R. Á., Delgado, R. C., Oliveira, L. J. C., Sarmento, A. P. 
Revista Brasileira de Geografia Física v.11, n.03 (2018) 745-757.

Meteorológica Mundial (OMM), em todos os pontos demarcados e registrados em planilha de campo, para posterior formulação e compilação de dados para análise e discussão.

A rede pluviométrica instalada no espaço urbano da cidade de Catalão-GO teve como objetivo principal a realização de acompanhamento das distribuições dos totais pluviométricos para o mapeamento e correlação da estrutura espacial das chuvas na cidade.

Salienta-se que os pluviômetros foram adquiridos por meio de parceria firmada entre a UFG/RC - Universidade Federal de Goiás, Regional Catalão - a Unidade Acadêmica
Especial Instituto de Geografia da UFG/RC e a Superintendência de Água e Esgoto (SAE) do referido Município, e foram destinados exclusivamente à pesquisa referente à precipitação na área urbana de Catalão. Assim sendo, cabe mencionar que foram instalados os pluviômetros referentes ao modelo Ville de Paris, com uma superfície receptora de $400 \mathrm{~cm}^{2}$, a $1,5 \mathrm{~m}$ acima da superfície do solo e de forma equitativa no espaço urbano para a coleta dos dados pluviométricos, de acordo com as orientações do INMET (Figura 2).

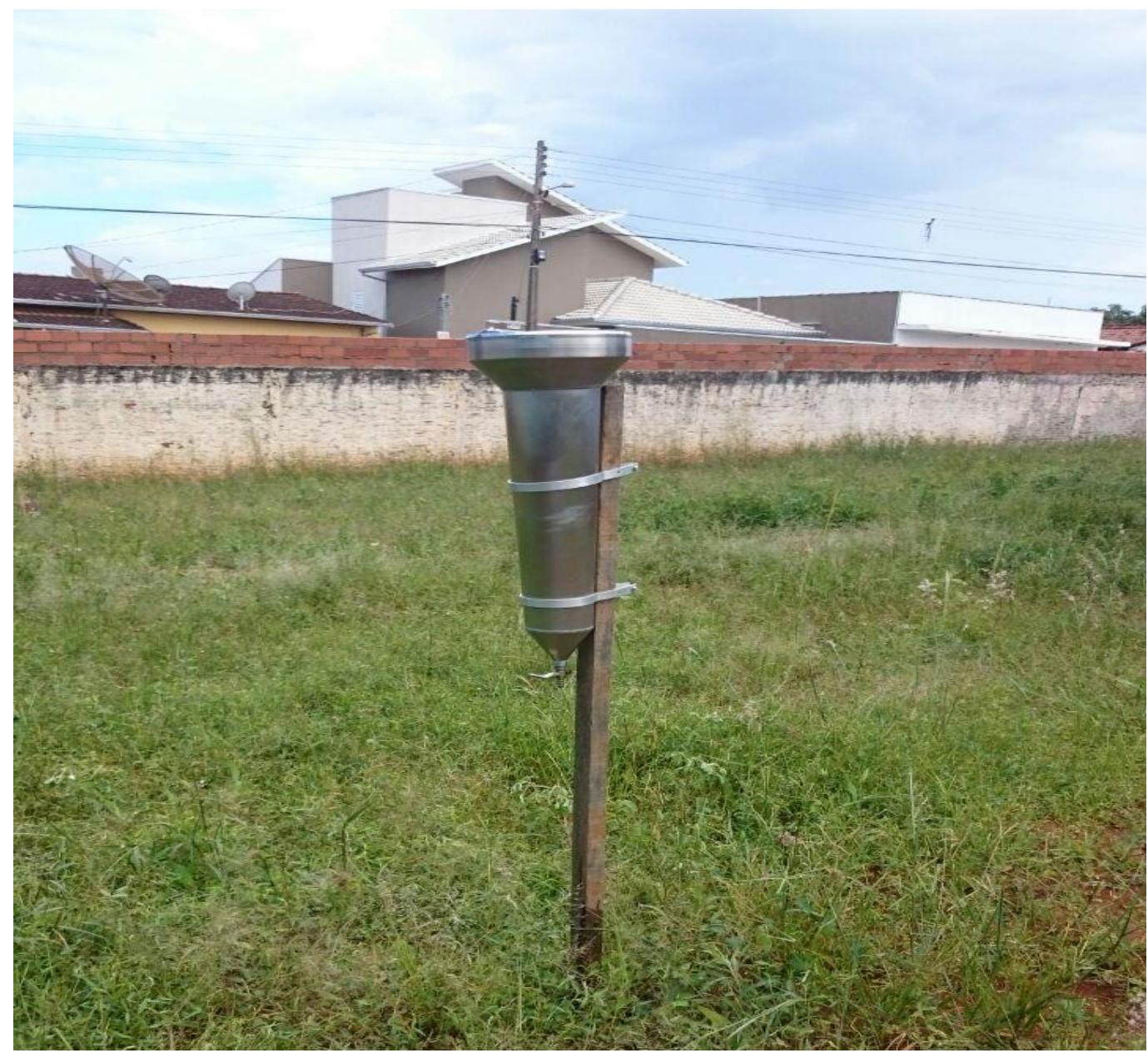

Figura 2. Exemplo de um dos pluviômetros tipo Ville de Paris instalados na malha municipal de CatalãoGO.

\section{Análise geoestatística}

Os pluviômetros e pluviógrafos espalhados pela superfície terrestre coletam informações precisas da chuva, no entanto esta é válida somente para uma pequena área localizada no entorno do dispositivo.
Para a escolha do melhor método espacial para a área urbana de Catalão, Goiás foi utilizado o método de interpolação espacial Krigagem Ordinária (KO), no programa ArcGIS 10.5, para analisar e calcular a distribuição espacial das chuvas na área urbana.

Silva, T. P., Rodrigues; R. Á., Delgado, R. C., Oliveira, L. J. C., Sarmento, A. P. 
Ao contrário de outros métodos de interpolação espacial, a krigagem estima uma matriz de covariância espacial que permite determinar os pesos atribuídos às diferentes amostras, a redundância dos dados, a vizinhança a ser considerada no procedimento inferencial e o erro associado ao valor estimado (Goovaerst, 1997).

$\mathrm{Na}$ Krigagem a variação espacial é quantificada por um semivariograma. $\mathrm{O}$ semivariograma é um gráfico de dispersão da semivariância versus distância dos pontos amostrados, sendo que a semivariância é uma medida de dispersão, a metade da variância.

Os valores da cota $\mathrm{z}$ são estimados em localizações espaciais $\left(\mathrm{x}_{\mathrm{j}}, \mathrm{y}_{\mathrm{j}}\right)$ não-observadas, sem a necessidade de se conhecer a média estacionária $\mu$, a partir de uma combinação linear dos valores de um subconjunto amostral local. A condição estabelecida no estudo foi a soma dos ponderadores da $\mathrm{KO} \lambda_{\mathrm{i}}\left(\mathrm{x}_{\mathrm{j}}, \mathrm{y}_{\mathrm{j}}\right)$ fosse igual a 1 .

O estimador de KO é dado pela equação

1.

$z\left(x_{j}, y_{j}\right)=\sum_{i=1}^{n(j)} \lambda_{i}\left(x_{j}, y_{j}\right) * z\left(x_{i}, y_{i}\right)$

em que, $\mathrm{z}$ é a cota na posição $\left(\mathrm{x}_{\mathrm{j}}, \mathrm{y}_{\mathrm{j}}\right)$ e $\lambda_{\mathrm{i}}\left(\mathrm{x}_{\mathrm{j}}, \mathrm{y}_{\mathrm{j}}\right)$, o ponderador da $\mathrm{KO}$, para a cota especificada.

Foi realizada a verificação da dependência espacial pelo cálculo de semivariograma, em que foram ajustados e testados os modelos teóricos exponencial, esférico e gaussiano, com objetivo de verificar qual o modelo que melhor representa a distribuição de cada variável estudada de acordo com as equações e os três modelos físicos apresentados por Silva et al. (2016).

Para validar e verificar o desempenho dos modelos na estimativa das variáveis foram utilizados os seguintes parâmetros (Willmott, 1982; Cambardella et al., 1994; Silva et al., 2016): o menor grau de dependência espacial (GDE \%), maior coeficiente de determinação $\left(\mathrm{r}^{2}\right)$, maior índice de concordância de Willmott (d), menor erro padrão da estimativa (EPE) e menor viés médio (VM).

\section{Resultados e discussão}

Estes procedimentos são explicitados e fundamentados pelas equações $2,3,4,5$ e 6 . $\mathrm{O}$ GDE é calculado pela seguinte equação:

$$
\mathrm{GDE}=\frac{\mathrm{C}_{0}}{\mathrm{C}_{0}+\mathrm{C}} \times 100
$$

em que $\mathrm{C}_{0}=$ intercepto ou efeito pepita e $\mathrm{C}_{0}+$ $\mathrm{C}=$ patamar.

O GDE pode ser classificado em: valores $\leq 25 \%$ os dados possuem forte dependência espacial, GDE entre 25 e $75 \%$ mostram moderada dependência espacial, GDE $\geq 75 \%$ possuem fraca dependência espacial e GDE igual a $100 \%$ a variável é espacialmente independente, ou seja, apresenta semivariograma com efeito pepita puro (EPP).

Depois de constatada a dependência espacial, a Krigagem Ordinária foi aplicada para estimar as variáveis em locais não amostrados, possibilitando a realização dos mapas. As análises do semivariograma e Krigagem foram realizadas no software ArcGIS 10.5 .

$$
\mathrm{r}^{2}=\frac{\sum_{\mathrm{i}=1}^{\mathrm{n}}\left(\mathrm{P}_{\mathrm{i}}-\overline{\mathrm{O}}\right)^{2}}{\sum_{\mathrm{i}=1}^{\mathrm{n}}\left(\mathrm{O}_{\mathrm{i}}-\overline{\mathrm{O}}\right)^{2}}
$$

$\mathrm{VM}=\frac{\sum_{\mathrm{i}=1}^{\mathrm{n}}\left(\mathrm{P}_{\mathrm{i}}-\mathrm{O}_{\mathrm{i}}\right)}{\mathrm{N}}$

$\mathrm{EPE}=\sqrt{\frac{\sum\left(\mathrm{O}_{\mathrm{i}}-\mathrm{P}_{\mathrm{i}}\right)^{2}}{\mathrm{n}-1}}$

$$
\mathrm{d}=1,0-\frac{\sum_{\mathrm{i}=1}^{\mathrm{N}}\left(\mathrm{P}_{\mathrm{i}}-\mathrm{O}_{\mathrm{i}}\right)^{2}}{\sum\left(\left|\mathrm{P}_{\mathrm{i}}-\overline{\mathrm{O}_{\mathrm{i}}}\right|+\mid \mathrm{O}_{\mathrm{i}}-\overline{\mathrm{O}_{\mathrm{i}}}\right)^{2}}
$$

em que, Pi é o iésimo valor estimado da chuva $(\mathrm{mm})$, Oi é o iésimo valor observado da chuva (mm), O é a média dos valores observados $(\mathrm{mm})$ e $\mathrm{N}$ é o número de dados analisados.

Na Figura 3 e 4, foram realizadas as comparações estatísticas dos dados de chuva 
Revista Brasileira de Geografia Física v.11, n.03 (2018) 745-757.

para os vinte pontos de pluviômetros para o ano de 2015. Baseado nos parâmetros estatísticos, o ano de 2015 foi o mais inconsistente pontualmente quando comparado com os dados observados das estações convencional e automática do INMET, onde, o $\mathrm{r}^{2}$ variou de $93 \%$ comparado com a convencional a $64 \%$ com a automática. Os erros foram maiores neste ano também, apresentando alta dispersão dos dados com VM para convencional de $-146 \mathrm{~mm}$ e EPE de 21,13 mm, já a automática os erros foram menores com VM de 13,41 mm e EPE de 49,72 $\mathrm{mm}$.

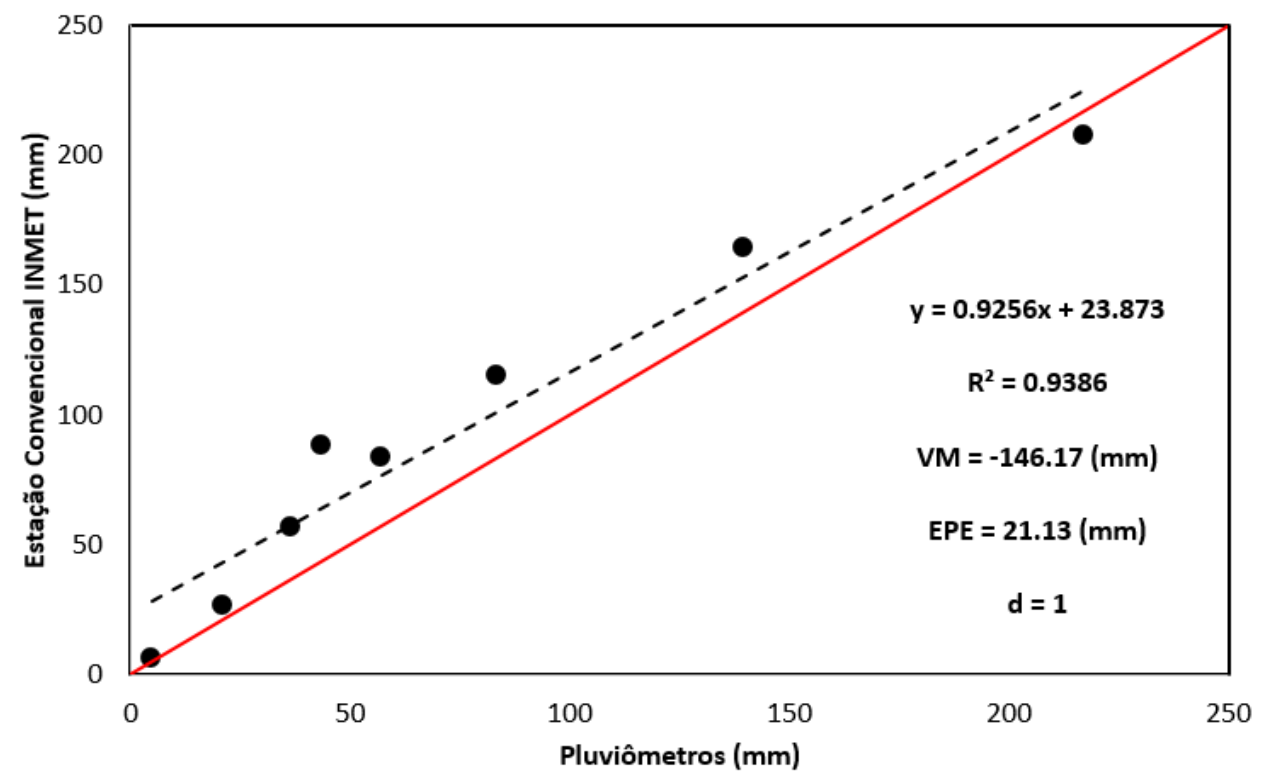

Figura 3. Análise estatística entre os dados observados da convencional com os dados estimados pela rede de pluviômetros para o ano de 2015.

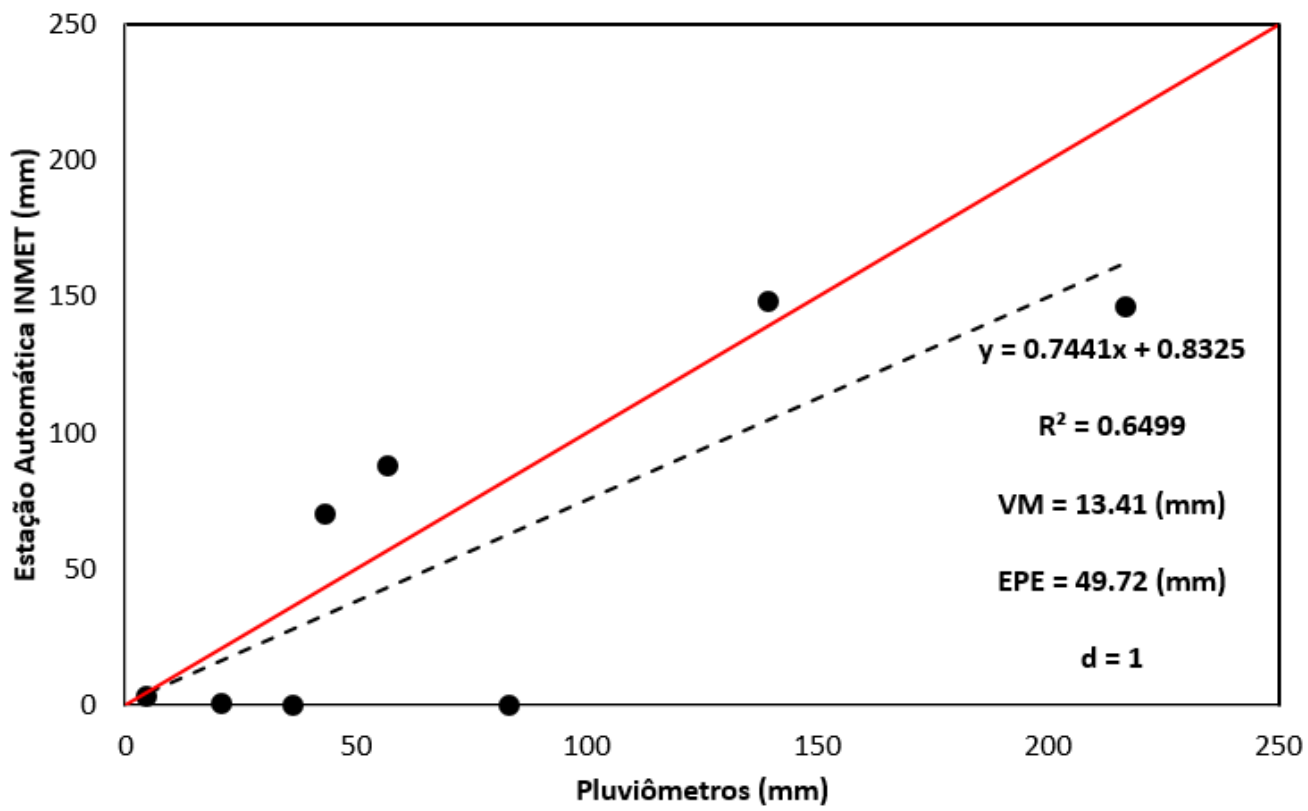

Figura 4. Análise estatística entre os dados observados da automática com os dados estimados pela rede de pluviômetros para o ano de 2015.

Silva, T. P., Rodrigues; R. Á., Delgado, R. C., Oliveira, L. J. C., Sarmento, A. P. 
Revista Brasileira de Geografia Física v.11, n.03 (2018) 745-757.

Para o ano de 2016 os resultados encontrados foram mais consistentes pontualmente para chuva na região, onde o $\mathrm{r}^{2}$ foi superior a 95\% para ambas estações (Figura 5 e
6). O índice d foi igual a 1 para ambas estações, o que indica baixa dispersão dos dados.

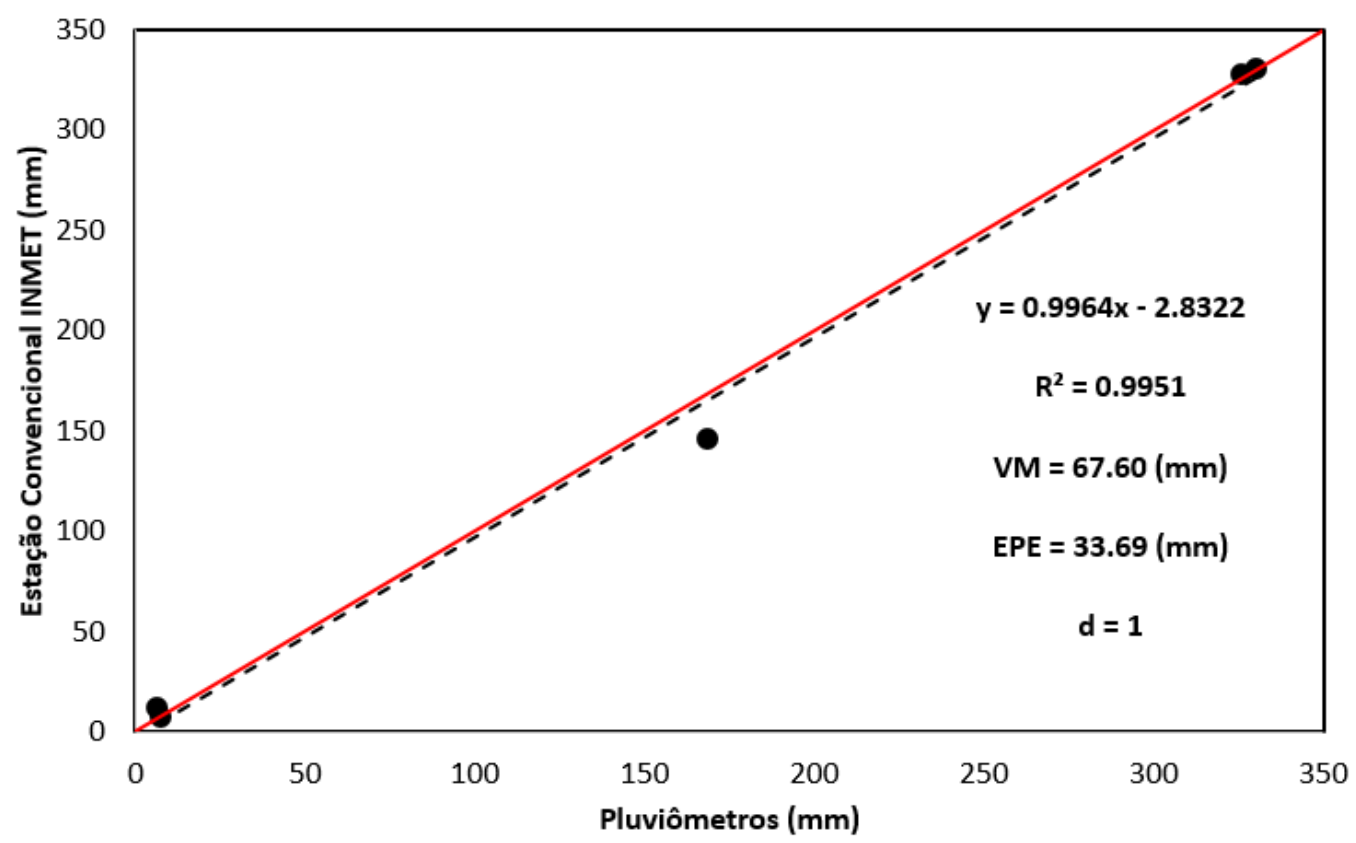

Figura 5. Análise estatística entre os dados observados da convencional com os dados estimados pela rede de pluviômetros para o ano de 2016.

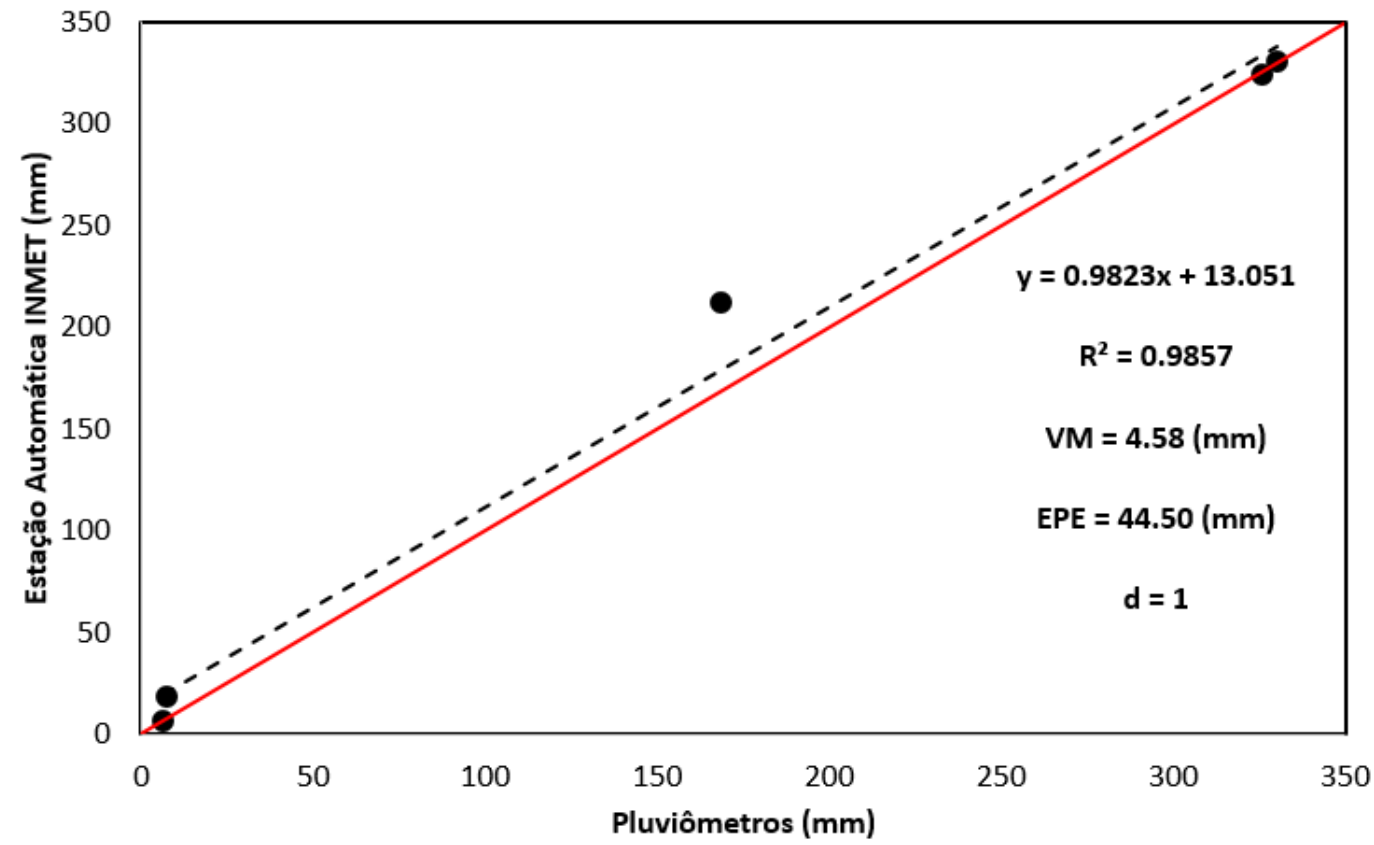

Figura 6. Análise estatística entre os dados observados da automática com os dados estimados pela rede de pluviômetros para o ano de 2016.

Depois de explorados estatisticamente, os dados foram submetidos à aplicação do semivariograma, de forma a analisar a dependência espacial da chuva, bem como obter os parâmetros do semivariograma juntamente como o grau de dependência espacial para cada modelo estudado (Tabela 2). Os ajustes dos

Silva, T. P., Rodrigues; R. Á., Delgado, R. C., Oliveira, L. J. C., Sarmento, A. P. 
Revista Brasileira de Geografia Física v.11, n.03 (2018) 745-757.

semivariogramas foram feitos de maneira interativa com o aplicativo do ArcGIS 10.5

O melhor GDE encontrado foi de $4,72 \%$, representando forte dependência espacial para o ano de 2016, em que se ajustou um modelo Exponencial. Para este mesmo ano, outros dois modelos foram significativos o Gaussiano e Esférico 36,29\% e 7,52\% (Tabela 2).

Os piores resultados foram encontrados para o ano de 2015, onde ambos modelos apresentaram Efeito Pepira Puro, o que inviabiliza a utilização de KO. Foi possível gerar os mapas espacializados dos três modelos espaciais estudados da chuva para Catalão, Goiás para o ano de 2016.

Resultados semelhantes utilizando a geoestatística foram encontrados por Varella et al. (2012), em que o melhor ajuste foi para o modelo gaussiano no experimento de brix com cana-de-açúcar e Castro et al. (2010), em que constatou-se que a krigagem é o melhor método de estimativa para as variáveis precipitação, evapotranspiração potencial, evapotranspiração real, deficiência hídrica, excedente hídrico e disponibilidade hídrica para o Espírito Santo. Panosso et al. (2008) trabalhou com Tabela 2. Parâmetros de ajustes do semivarioagrama e sua classe de acordo com o GDE encontrado.

\begin{tabular}{cccccc}
\hline Anos & $\mathrm{C}_{0}$ & $\mathrm{C}_{0}+\mathrm{C}$ & $\mathrm{GDE}(\%)$ & Classe & Modelos \\
\hline \multirow{2}{*}{2015} & 17976.02 & 17976.02 & 100 & EPP & Esférico \\
& 17976.02 & 17976.02 & 100 & EPP & Exponencial \\
& 17976.02 & 17976.02 & 100 & EPP & Gaussiano \\
\hline \multirow{2}{*}{2016} & 184.29 & 2452.26 & 7.52 & Forte & Esférico \\
& 126.57 & 2681.32 & 4.72 & Forte & Exponencial \\
& 903.41 & 2489.19 & 36.29 & Moderada & Gaussiano
\end{tabular}

$\mathrm{C}_{0}=$ efeito pepita; $\mathrm{C}_{0}+\mathrm{C}=$ patamar; $\mathrm{a}=$ alcance; $\mathrm{GDE}=$ Grau de Dependência Espacial; EPP $=$ Efeito Pepita Puro.

A Figura 7, 8 e 9 mostra o recorte da área urbana de Catalão, Goiás, onde na adoção dos modelos exponencial, gaussiano e esférico as maiores chuvas para este ano de 2016 concentram-se na orientação norte $(\mathrm{N})$ com total anual chegando acima de $900 \mathrm{~mm}$. As menores taxas pluviométricas são encontradas na faixa central da área urbana com total anual oscilando entre $810 \mathrm{~mm}$ a $830 \mathrm{~mm}$. As precipitação anuais para ambos os modelos geoestatística em dois experimentos com a cultura da cana-de-açúcar, analisando a variabilidade espacial da emissão de $\mathrm{CO}_{2}$ e umidade do solo que são desprovidos de vegetação.

Em um estudo de seca no Estado do Tocantis Gois et al. (2015) identificaram que ambos modelos exponencial e esférico foram capazes de identificar espacialmente o fenômeno nesta região.

Outro estudo recente de interpolação espacial utilizando Krigagem Ordinária no Estado do Mato Grosso os autores Ramos et al. (2017) identificaram que o modelo exponencial se adequou mais ao período chuvoso, no entanto, para variável temperatura o modelo gaussiano foi o mais representativo.

Também no mesmo Estado em Goiás, os autores Alves e Vecchia (2011) utilizaram outros métodos de estimativa da precipitação (mínima curvatura, inverso quadrático da distância e krigagem), onde os métodos mínima curvatura e inverso quadrático da distância foram melhores que a krigagem em uma escala muito maior do que a malha urbana de Catalão utilizada neste trabalho. 
Revista Brasileira de Geografia Física v.11, n.03 (2018) 745-757.

ambos os estudos na espacialização da chuva para os modelos no ano de 2016 se deve aos pontos dos pluviômetros instalados não possuírem uma topografia complexa com altitude média de aproximadamente 900 metros.

Rodrigues e Castro (2008) por meio de uma rede de pluviômetros instalados na área urbana de Araguari (MG) observaram que a estrutura espacial das chuvas é condicionada pelo deslocamento dos sistemas atmosféricos: a Frente Polar e as Linhas de Instabilidade Tropical, as quais no decorrer dos meses possuem diferentes direções de deslocamento o que pode conduzir a esta distribuição geográfica das chuvas em Catalão-GO nos diferentes pluviômetros.

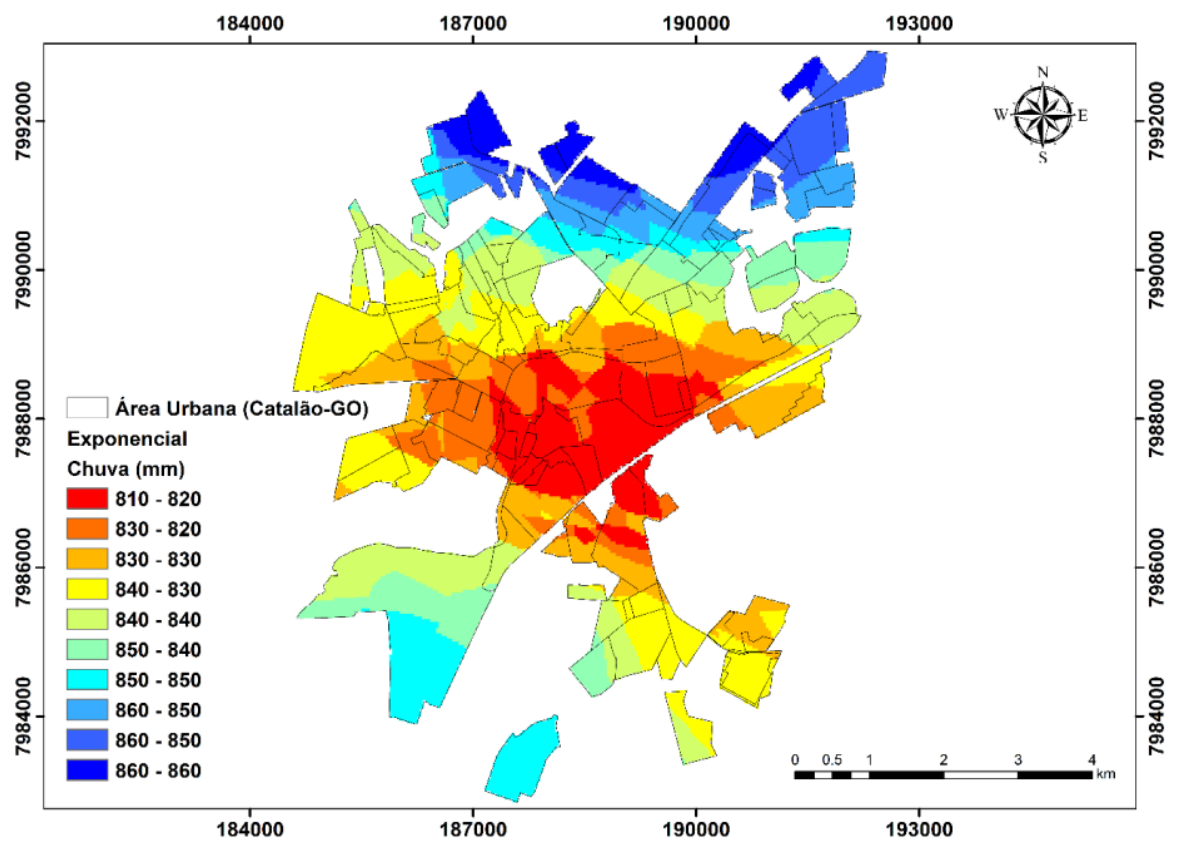

Figura 7. Análise espacial da chuva pelo modelo interpolado exponencial para o ano de 2016.

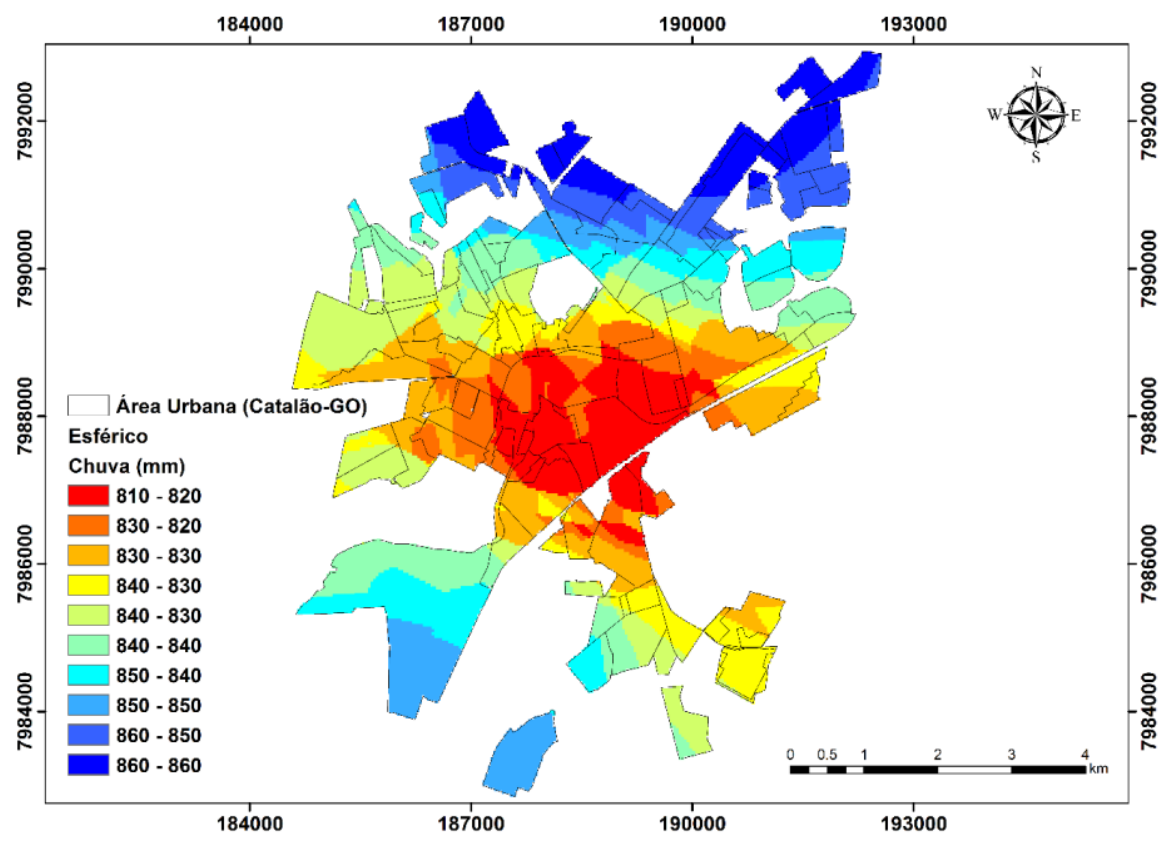

Figura 8. Análise espacial da chuva pelo modelo interpolado esférico para o ano de 2016.

Silva, T. P., Rodrigues; R. Á., Delgado, R. C., Oliveira, L. J. C., Sarmento, A. P. 


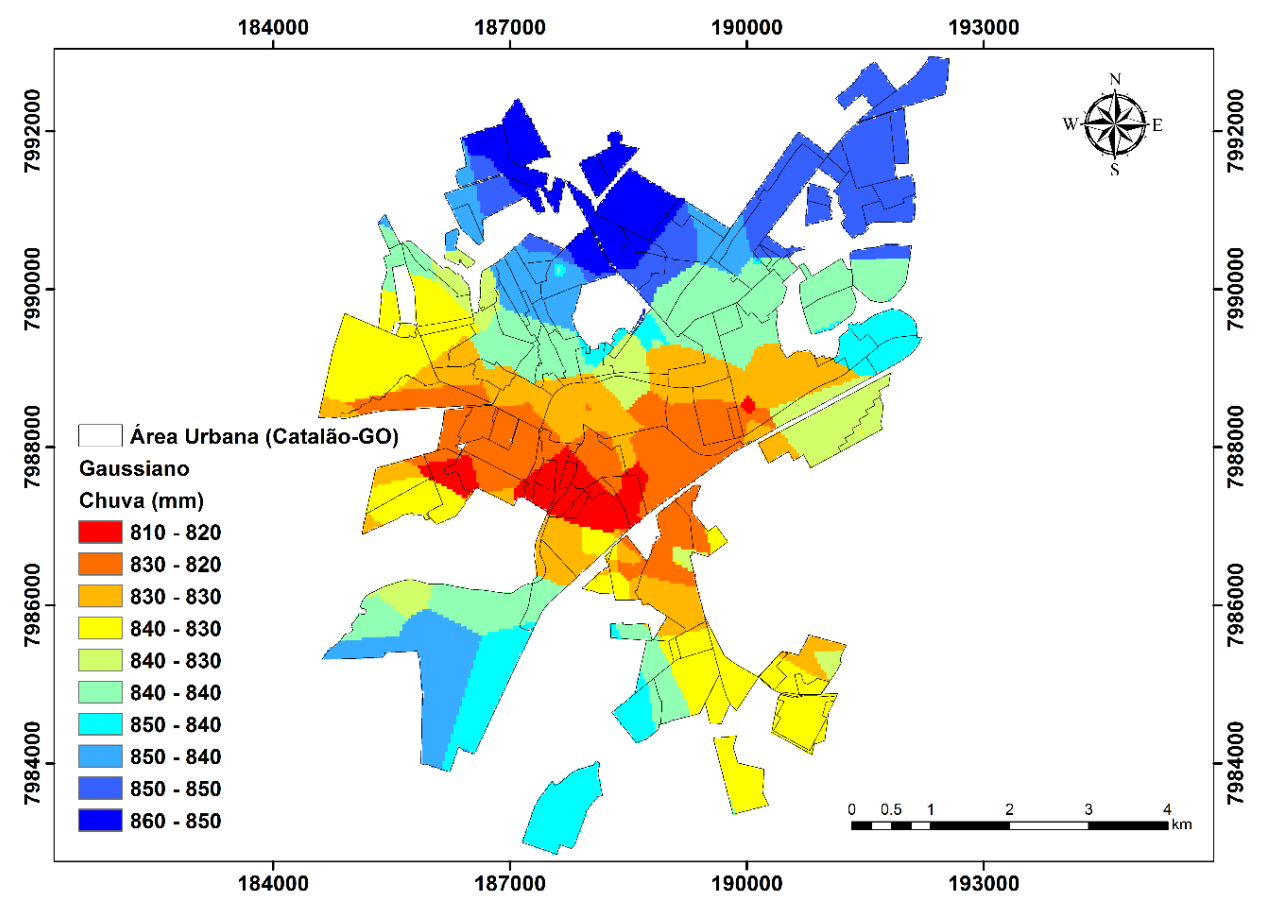

Figura 9. Análise espacial da chuva pelo modelo interpolado gaussiano para o ano de 2016.

Os bairros, periféricos do recorte espacial, extremos sul e norte, como evidenciado na Figura 6, 7 e 8, apresentaram ao longo da pesquisa maiores volumes precipitados. Vale ressaltar que pontos como P01 - Escola Municipal Natália Safatle Soares localizada bairro Evelina Nour, P07 Elevatório de Esgoto do bairro Leblon e P10 Estação de Tratamento de Água do DIMIC (Distrito Minero Industrial de Catalão) também apresentam menores índices de adensamento geográfico, menor área impermeabilizada e consequentemente menores barreiras físicas para o deslocamento de ventos. Os maiores valores precipitados também foram encontrados no pluviômetro P06 (ETA Ipanema) 920,70 mm, onde a altitude média do local é de 906 metros.

As áreas centrais da cidade de Catalão, pontos Estação Meteorológica Convencional INMET, no bairro Pio Gomes e P14 - Labibe Fayad no bairro JK, foram os pontos com menores índices pluviométricos. Essas áreas estão em regiões com maior adensamento geográfico, maior área de impermeabilização e com mais barreiras físicas para o deslocamento de ventos, bem como, estão nas áreas de menor altitude do espaço urbano de Catalão. A menor chuva foi encontrada no pluviômetro P16 (Setor Paraíso) 766,40 mm.
Estas regiões de maiores e menores índices pluviométricos encontrados podem estar associados ao aumento das células convectivas, em detrimento ao aumento da temperatura da superfície como o trabalho de Delgado et al. (2012), onde os autores ao estudarem a influência da mudança da paisagem, a partir de dados TM em Cruzeiro do Sul, AC, constataram o crescimento das áreas antropizadas entre os anos de 2005 a 2010. Outro resultado importante encontrado por estes pesquisadores foi o aumento da temperatura da superfície em áreas antropizadas com valores superiores a $40^{\circ} \mathrm{C}$, resultando no aumento da precipitação de 17,63 mm/ano (1971-1990) e alcançando um máximo de $30,48 \mathrm{~mm} /$ ano nos anos de 1993 a 2002.

\section{Conclusão}

A KO pôde ser aplicada para toda malha urbana de catalão para o ano de 2016. Já o ano de 2015 apresentou alta dependência espacial, o que impossibilita a sua espacialização.

Através deste estudo, a técnica utilizada de geoestatística proporcionou resultados satisfatórios quando da dependência espacial da chuva para os anos estudados.

Silva, T. P., Rodrigues; R. Á., Delgado, R. C., Oliveira, L. J. C., Sarmento, A. P. 
Revista Brasileira de Geografia Física v.11, n.03 (2018) 745-757.

As regiões de maiores e menores adensamentos geográficos influenciaram diretamente nos volumes precipitados em Catalão, Goiás.

\section{Agradecimentos}

$\mathrm{O}$ autor agradece à Fundação de Amparo à Pesquisa do Estado de Goiás (FAPEG) pela concessão da bolsa de Iniciação Científica, ao CPTPI - Centro de Pesquisas Tecnológicas e Processamento de Imagem da Universidade Federal de Goiás - Regional Catalão, pela infraestrutura disponível no desenvolvimento da pesquisa, e à Superintendência de Água e Esgoto pelo financiamento dos pluviômetros.

\section{Referências}

Alves, E. D. L., Vecchia, F. A. S., 2011. Análise de diferentes métodos de interpolação para a precipitação pluvial no Estado de Goiás. Acta Scientiarum. Human and Social Sciences 33, 193-197.

Cambardella, C. A., Moorman, T. B., Novak, J. M., Parkin, T. B., Karlen, D. L., Turco, R. F., Konopka, A. E., 1994. Field scale variability of soil properties in central Iowa soil. Soil Science Society of America Journal 58, 1501-1511.

Castro, F. D. S., Pezzopane, J. E., Cecílio, R. A., Pezzopane, J. R., Xavier, A. C., 2010. Avaliação do desempenho dos diferentes métodos de interpoladores para parâmetros do balanço hídrico climatológico. Revista Brasileira de Engenharia Agrícola e Ambiental 14, 871-880.

Delgado, R. C., de Oliveira Junior, J. F., Gois, G., Lyra, G. B., 2014. Cenários Climáticos da Radiação Solar Global Baseados no Modelo Regional HadRM3 para o Estado do Acre. Floresta e Ambiente 21, 417-428.

Delgado, R. C., Souza, L. P., Silva, I. W. R., Pessôa, C. S., Gomes, F. A., 2012. Influência da mudança da paisagem amazônica no aumento da precipitação em Cruzeiro do Sul, AC. Enciclopédia Biosfera 8, 665-674.
Fengqing, J., Cheng, Z., Guijin, M., Ruji, H., \& Qingxia, M., 2005. Magnification of flood disasters and its relation to regional precipitation and local human activities since the 1980s in Xinjiang, Northwestern China. Natural Hazards 36, 307-330.

Gois, G., Delgado, R. C., Oliveira-Júnior, J. F., 2015. Modelos teóricos transitivos aplicados na interpolação espacial do Standardized Precipitation Index (SPI) para os Episódios de El Niño forte no estado do Tocantins, Brasil. Irriga 20, 371-387.

Gondim, R. S., Castro, M. D., Evangelista, S. D. M., Teixeira, A. D. S., Fuck Júnior, S. D. F., 2008. Mudanças climáticas e impactos na necessidade hídrica das culturas perenes na Bacia do Jaguaribe, no Estado do Ceará. Pesquisa Agropecuária Brasileira 43, 1657 1664.

Goovaerst, P., 1997. Geostatistics for natural resources evaluation. New York: Oxford University Press, 481.

IBGE - Instituto Brasileiro de Geografia e Estatística. Disponível em: < http://cidades.ibge.gov.br/xtras/perfil.php?c odmun=520510>. Acesso: 08 ago. 2017.

IBGE, Divisão Territorial do Brasil e Limites Territoriais. Instituto Brasileiro de Geografia e Estatística (IBGE). 2008. Disponível em: ftp://geoftp.ibge.gov.br/organizacao_territor ial/divisao_territorial. Acesso: 12 jan. 2015.

Instituto Nacional De Meteorologia (INMET). Disponível em: http://www.inmet.gov.br/. Acesso: 02 fev. 2015.

Mendonça, F., 2003. Clima e Planejamento Urbano em Londrina. Proposição metodológica e de intervenção urbana a partir do estudo do campo termohigrométrico. In: Mendonça, F., Monteiro, C. A. F. Clima Urbano. São Paulo: Contexto, 2003.

Panosso, A. R., Pereira, G. T., Marques Júnior, J., La Scala Júnior, N., 2008. Variabilidade espacial da emissão de $\mathrm{CO}_{2}$ em latossolos

Silva, T. P., Rodrigues; R. Á., Delgado, R. C., Oliveira, L. J. C., Sarmento, A. P. 
sob cultivo de cana-de-açúcar em diferentes sistemas de manejo. Engenharia Agrícola 28, 227-236.

Pereira, M. D. B., 2016. Eventos pluviométricos extremos na cidade de João Pessoa: análise dos eventos dos dias 16 e 17 de Abril de 2016. Revista de Geociências do Nordeste 2, 575-584.

Polade, S. D., Pierce, D. W., Cayan, D. R., Gershunov, A., Dettinger, M. D., 2014. The key role of dry days in changing regional climate and precipitation regimes. Scientific reports $4,4364$.

Ramos, H. C., Dallacort, R., da Silva Neves, S. M. A., Dalchiavon, F. C., Santi, A., Vieira, F. F., 2017. Precipitação e temperatura do ar para o Estado de Mato Grosso utilizando krigagem ordinária. Revista Brasileira de Climatologia 20, 211-233.

Rodrigues, R. A., Castro, S. S. A., 2008. Estrutura Espacial das Chuvas na Cidade de Araguari (MG) Durante a Estação Chuvosa 2001-2005. Revista Geográfica Acadêmica 2, 43-55.

Rodrigues, R. A., Oliveira, G. A., Faria, A. L. L., Oliveira Júnior., 2009. Caracterização Climática no Entorno da Usina Hidroelétrica Serra do Facão (GO). In: Torres, F. T. P., Dagnino, R. S., Oliveira Júnior, A. (Orgs.). Contribuições Geográficas, 521-542.

Rodrigues, R. A., Oliveira, G. A., Faria, A. L. L., Oliveira Júnior, A., Delgado, R. C., 2012. Determinação de Regiões climaticamente homogêneas no Estado de Goiás. In: Assis, A. A. F., Faria, A. L. L., (Orgs.). O onde e o quando: espaço e memória na construção da história e da geografia, 274-289.

Santana, M. F., Lisboa, H. C. K. J., Delgado, R. C., Oliveira Júnior, J. F., Gois, G., Rodrigues, R. Á., Souza, L. P., 2015. Desempenho do Modelo HadRM3 Baseado em Geoestatística para Subsidiar o Zoneamento da Heveicultura na Amazônia. Nativa 3, 83-88.

Santos C., R., Amorim, M. C. D. C. T., 2015. Características do clima urbano em
Presidente Prudente/SP a partir de dados de temperatura e umidade relativa do ar e técnicas de sensoriamento remoto. Revista do Departamento de Geografia 28, 39-64.

Silva, C. S., Pereira, M. G., Delgado, R. C., Silva, E. V., 2016. Spatialization of soil chemical and physical attributes in an agroforestry system, Seropédica, Brazil. Cerne 22, 407-414.

Sun, W., Zhu, Y., Huang, S., Guo, C., 2015. Mapping the mean annual precipitation of China using local interpolation techniques. Theoretical and applied climatology 119, 171-180.

Trindade, A. L. F., de Oliveira, P. T. S., Anache, J. A. A., Wendland, E., 2016. Variabilidade espacial da erosividade das chuvas no Brasil. Pesquisa Agropecuária Brasileira 51, 19181928.

Varella, C. A. A., Barros, V. R., Baesso, M. M., 2012. Mapeamento da variabilidade espacial do brix em cana-de-açúcar. Engenharia na Agricultura 20, 112-117.

Viana, S. M., Amorim, M. C. D. C. T., 2009. O clima urbano em Teodoro Sampaio/SP: Episódios de verão. Revista Brasileira de Climatologia 5, 41-54.

Willmott, C. J., 1982. Some comments on the evaluation of model performance. Bulletin American Meteorological Society 63, 13091313.

Xavier, A. C., King, C. W., Scanlon, B. R., 2016. Daily gridded meteorological variables in Brazil (1980-2013). International Journal of Climatology 36, 2644-2659.

Zanella, M. E., Sales, M. C. L., 2016. Impactos pluviais em Fortaleza-CE na perspectiva do sistema clima urbano (Rainfall impacts in Fortaleza-CE from the perspective of the urban climate system-hydro-meteorological subsystem). Revista Brasileira de Geografia Física 9, 2290-2300.

Silva, T. P., Rodrigues; R. Á., Delgado, R. C., Oliveira, L. J. C., Sarmento, A. P. 